\title{
Tibial plateau fractures in Belgium: epidemiology, financial burden and costs curbing strategies
}

\author{
* Michiel Herteleer, MD 1,2,; * Celien Van Brandt, MD3; Cindy Vandoren, MSc4; Stefaan Nijs, MD PhD \\ 2,3; Harm Hoekstra, MD PhD FEBS2,3 \\ 1 Department of Orthopaedics and Traumatology, Unimedizin Mainz, Mainz, Germany \\ ${ }_{2}$ KU Leuven - University of Leuven, Department of Development and Regeneration, Leuven, Belgium \\ 3 University Hospitals Leuven, Department of Trauma Surgery, Leuven, Belgium \\ 4 University Hospitals Leuven, Management Information and Reporting, Leuven, Belgium \\ * both authors contributed equally \\ Corresponding Prof. Harm Hoekstra, harm.hoekstra@uzleuven.be \\ Corresponding address: University Hospitals Leuven, Department of Trauma Surgery, Herestraat 49, \\ B-3000 Leuven, Belgium
}

Declarations of interest: none

Funding: This research did not receive any specific grant from funding agencies in the public, commercial, or not-for-profit sectors.

Ethical approval: Ethical approval for this study was granted by the Research Ethics Committee UZ/KU Leuven and registered as MP009477.

\author{
ABBREVIATIONS \\ TPF tibial plateau fractures \\ TKA total knee arthroplasty \\ LOS length-of-stay \\ UHL University Hospitals Leuven \\ ROS rate of surgery \\ NIHDI National Institute for Health and Disability Insurance
}

KEYWORDS

Knee, Tibial Plateau Fracture, Economic burden, Length-of-stay

LEVEL OF EVIDENCE: 4 


\section{ABSTRACT \\ Purpose}

We describe the incidence of tibial plateau fractures and the evolution of its management and financial burden in Belgium, perform a similar audit at University Hospitals Leuven, and define strategies to curb the increasing cost.

\section{Methods}

National data on tibial plateau fractures was collected from the NIHDI and compared to our performance. Several clinical parameters such as age, sex, treatment modality and length-of-stay were included. The total healthcare costs are considered as the sum of hospitalization costs and ambulatory care costs.

\section{Results}

Between 2006 and 2018, a total number of 35,226 tibial plateau fractures were diagnosed in Belgium and 861 in our center. The incidence increased $41 \%$ over time (mean 25/100,000 persons per year). The mean rate of surgery in Belgium was $37 \%$ and slightly decreased over time, due to a larger increase of nonoperatively treated tibial plateau fractures. The rate of surgery at the UHL was $49 \%$. Surprisingly, the average cost per patient was equal for operatively and non-operatively treated patients in Belgium, and driven by the length-of stay.

\section{Conclusion}

Since length-of-stay is the main driver of the total healthcare costs of tibial plateau fractures, guidelines on appropriate length-of-stay can help to decrease variability and curb the total healthcare costs, particularly of the non-operatively treated patients. Our performance was in line with this. 


\section{INTRODUCTION}

Tibial plateau fractures (TPF) account approximately $1 \%$ of all fractures in adults and up to $8 \%$ of all fractures in elderly [1, 2]. Wennergren et al. reported in 2018 an increasing incidence of TPF in Sweden with an annual incidence of 26.9 per 100,000 [3]. This increase accounted mainly elderly woman, whereas the incidence of men remained stable. These authors concluded that TPF are most often osteoporosis-related.

Although there is often a clear indication for operative management of TPF, the decision to operate should carefully be considered nevertheless. Morbidity increases with the age; malalignment, fracture related infection, non-union, and other postoperative complications, have far-reaching consequences, not only functionally but also financially $[4,5]$. At long term, post-traumatic osteoarthritis occurs in $25 \%$ to $45 \%$, with a 10-year likelihood of 3\%-7\% for total knee arthroplasty (TKA) and increase of total healthcare costs, subsequently [6].

We recently demonstrated that the rate of surgery for clavicular fractures in Belgium has almost doubled in 10 years, while the indication for this is still under debate [2]. In parallel, the total healthcare costs increased, due to hospitalization and honoraria, the latter mainly based on a fee-for-service principle [7]. Given the complexity of its management and financing, TPF pose a great health and financial risk. Hence, a better understanding of epidemiology of TPF is crucial to develop strategies to curb the financial burden. Therefore, we aimed to describe the incidence of TPF and the evolution of its management and financial burden in Belgium. In parallel, we examined the management and total healthcare costs of TPF in our hospital, compared it to the national data, and define strategies to curb the increasing cost. 


\title{
PATIENTS AND METHODS
}

This retrospective population-based study was administered by the Department of Traumatology at the University Hospitals Leuven (UHL, Belgium).

\section{Data Collection}

\begin{abstract}
Belgium
National data was collected from the Belgian National Institute for Health and Disability Insurance (NIHDIINAMI-RIZIV) [8]. NIHDI nomenclature numbers were used to retrieve data of all patients sustaining a TPF between 2006 and 2018. A nomenclature number is a numerical fee code that represents different diagnoses and management modalities. This code is mandatory to receive financial reimbursement. The data related to these codes is strictly anonymously and could not be linked to the individual patient. There were no exclusion criteria. The numbers 290485 and 290500 represents operative management of unicondylar and bicondylar TPF, respectively. The numbers 298362 and 298841 represents nonoperative management of TPF with and without fracture reposition, respectively. The number 290286 represents TKA. The nomenclature numbers were linked to the patients' age cluster (every age cluster encompassed one decade), sex, year of procedure, treatment modality (hospitalization or ambulatory care) and length-of-stay (LOS).
\end{abstract}

\section{University Hospital Leuven}

A total of 861 TPF treated in UHL between 2006 and 2018 were included. There were no exclusion criteria. The same NIHDI nomenclature numbers used to retrieve national data were used to retrieve UHL data. Using our electronic patient file system, the following patient data were collected: age, sex, date of injury, type of treatment (operative vs. non-operative), treatment modality (hospitalization or ambulatory care), LOS, date of TKA.

\section{Healthcare utilization and total healthcare costs}

The costs described in this paper relate to the Belgian healthcare financing system and are merely limited to costs of hospital-related care. The costs in this study are defined as the total reimbursements paid to the hospital by any party involved in financing the care for a specific patient either directly or indirectly. The total healthcare cost is considered as the sum of hospitalization costs and ambulatory care costs. Regarding the hospitalization costs, the LOS was multiplied by the national average day-based care fee (€437). Ambulatory costs are related to plaster application, exchange, removal, or other matters (e.g. wound care) related to TPF. Honoraria and material-related and pharmaceuticals costs were included in both cost categories (hospitalization and ambulatory care). The honoraria category consists of medical activity-related fees, based on a fee-for-service principle. Medical activities include consults, surgery and imaging. Materialrelated costs are cost of used implants and other materials (e.g. suture materials). Pharmaceuticals costs are all costs for received drugs and blood product. NIHDI provided data with regard to healthcare costs for TPF between 2008 and 2017, in the form of the annual mean cost. The healthcare costs were available for every distinct hospital stay at the UHL between 2008 and 2017. All costs analyzed were indexed.

\section{Statistical analysis}

Descriptive statistics were used to analyze the data in Excel. The LOS was compared using Chi-Square statistics and The Mann-Whitney $U$ test (Wilcoxon rank sum test) was used for comparing the hospitalization and ambulatory care costs. A significance level of $p<0.05$ was accepted for all tests. Incidences were calculated by dividing the number of TPF by the total number of persons in a 10-year age cluster and multiplied by 100,000 . National demographic information (i.e. population size per year per age category and 
sex) was obtained via Statbel [9]. The rate of surgery (ROS) was calculated by dividing the number of operatively treated TPF by the total number of diagnosed TPF in Belgium and UHL.

The 10-year likelihood ratio of a TKA after TPF in Belgium and UHL was the mean rate of TKA over 10 years calculated from 2008 onwards. The rate of TKA after a TPF was calculated as follows: (TKA/TPF)/(nonTKA/TPF)*100\%, differentiated towards operatively and non-operatively treated TPF. Due to privacy reasons, the IMA-AIM only allowed the NIHDI a random sampling of $1 / 40$ of the Belgians sustaining a TPF with an additional oversampling of $1 / 20$ for patients older than 65 .

\section{RESULTS}

\section{Fracture Incidence}

A total number of 35,226 TPF were diagnosed in Belgium between 2006 and 2018, of which 16,506 (47\%) occurred in men and $18,720(53 \%)$ in women (Table 1 ). The average incidence was $25 / 100,000$ persons per year between 2006 and 2018. There was a 41\% increase in incidence of TPF from 21/100,000 persons per year in 2006 to $29 / 100,000$ persons per year in 2018 . A $55 \%$ increase was observed in women $(21 / 100,000$ persons per year in 2006 and 32/100,000 persons per year in 2018), whereas $28 \%$ increase observed in men (21/100,000 persons per year in 2006 and 27/100,000 persons per year in 2018) (Figure 1). The age adjusted incidence of TPF in Belgium is displayed in Figure 2 and shows an increasing incidence in elderly, more pronounced in women than in men. The peak incidence of TPF in Belgium is displayed in Figure 3a. A total of 861 TPF were diagnosed at the UHL between 2006 and 2018 (2.4\% of all TPF in Belgium). 419 (49\%) TPF were diagnosed in men and $442(51 \%)$ in women. (Table 1). The peak incidence of TPF at the UHL was similar as for Belgium and lied between $40-49$ years of age in men and 50-59 years of age in women (Figure 3b).

\section{Fracture management}

The overall ROS in Belgium between 2006 and 2018 was 37\%, though slightly decreased (41\% in 2006 vs. $36 \%$ in 2018). This slight decrease was the result of a larger increase in number of non-operatively treated TPF compared to operatively treated fractures ( $68 \%$ vs. $31 \%$ increase, respectively). The ROS in relation to age was equal for male and female patients and displayed in Figure 4a.

The overall ROS at the UHL was $49 \%$ and in almost all age clusters higher as in Belgium, most pronounced between $50-79$ years old. (Figure $4 b$ )

\section{Total knee arthroplasty (TKA) after TPF}

For Belgium TKA after TPF was estimated using a random sampling as described. The 10 -year likelihood ratio for TKA after a TPF was $5 \%$ for operatively treated patients and $7 \%$ for non-operatively treated patients. Since 200823 (2.7\%) patients primary treated for a TPF at the UHL required a TKA: 2 patients got a TKA as primary treatment, 4 patients were initially treated non-operatively and 17 patients operatively. Nineteen out of 23 TKA (83\%) occurred in women. The 10 -year likelihood ratio of TKA after a TPF at the UHL was $4 \%$ in operatively treated patients and $1 \%$ in non-operatively treated patients.

\section{Healthcare utilization and related costs}

Table 2 shows the number of hospitalizations and ambulatory care in Belgium and UHL between 2008 and 2017. Between 2008 and 2017 there was predominantly an increase in ambulatory care of TPF in Belgium (Figure 5a), mainly due to increase of non-operatively treated patients (Figure 5b). In fact, the hospitalization rate of non-operatively treated patients decreased from $27 \%$ to $16 \%$. In parallel, we observed an increase in ambulatory care of non-operatively treated patients at the UHL (Figure $5 \mathrm{c}$ ). The number of hospitalization of operatively treated patients in Belgium increased with 15\% (from 974in 2008 to 1.120 in 2017). 
Table 2 displays the LOS of TPF patients in Belgium and at the UHL between 2008 and 2017. In contrast with the decrease in hospitalization rate of non-operatively treated patients (Figure 5b), we observed an increase in LOS of these patients in Belgium (Figure 6a). The LOS of operatively treated patients decreased over time however. There was a clear linear relation between the LOS and age in both operatively and non-operatively treated patients (Figure 6b).

The total healthcare costs for TPF in Belgium increased 20\% between 2008 and 2017 and were calculated on $€ 121,862,586$ ( 24,341 patients) of which $78 \%$ accounted operatively treated patients. The majority of these cost were related to hospitalization and honoraria. Implants accounted only $6 \%$ of the total healthcare costs. The increase of total healthcare cost at the UHL could also account mainly to the operatively treated patients. In contrast, the total healthcare cost for TPF at the UHL in the same period was $€ 4,071,181$ (659 patients), which was $3.3 \%$ of the total healthcare costs for TPF in Belgium. 


\section{DISCUSSION}

The primary aim of this study was to describe the incidence of TPF and the evolution of its management and financial burden in Belgium. Subsequently, we performed an audit at the UHL by comparing our performance on TPF to the national data, and define strategies to curb the increasing cost.

The NIHDI database provides a unique, population-covering data-set regarding the incidence and treatment of fractures in a population. The incidence of $25 / 100.000$ persons per year reported in our study is in line with the 26.9/100.000 persons per year reported by the Swedish Fracture Registry [3]. In the current retrospective study we found that over time there was an increasing incidence of TPF, however a decrease in ROS. We hypothesize that the reason for this is threefold: first there is an increasing number of osteoporotic fractures which are currently detected due to an increased awareness and higher quality imaging techniques such as CT and MRI. Secondly we believe that these fractures are often minimally displaced fractures and do not necessary require operative treatment. Lastly, frail elderly patients often have a significant number of comorbidities combined with reduced mobility, which results in a more wait and see attitude. However, as age is not an independent risk facture and elderly patients are not prone to infection $[6,10,11]$. As the used database is an insurance and cost oriented database, composed and controlled by the government it unfortunately does not allow us to diversify into supplementary data that is of interest to the trauma surgeon (e.g. fracture pattern, smoking status, open or closed fractures, etc.).

Court-Brown described the incidence of TPF as bimodal in male and female patients [12]. This bimodal distribution was neither observed in the Belgian nor in UHL population, wherein we saw a more unimodal like incidence distribution in women with increasing incidences in the elderly women. A more horizontal incidence curve was shown in men (Figure 2). Our results are comparable and in line with the registry studies of Sweden and Canada $[3,13]$. The increasing incidence in elderly women contributes to the hypothesis that in the elderly, these fractures are osteoporosis related fragility fractures. In Belgium, approximately $21 \%$ of all non-operatively treated patients were hospitalized, whereas $28 \%$ of the non-operatively treated UHL patients were hospitalized. This high hospitalization rate of non-operatively treated patients further supports the osteoporosis fragility fracture hypothesis. In our experience we see that elderly patients, who often live independently until old age, suddenly loose there self-sufficiency after sustaining such a fracture. As a consequence, they need to be admitted due to social reasons until an appropriate living environment for their discharge can be organized.

$63 \%$ of all TPF in Belgium were treated non-operatively. This is $14 \%$ more than the $59 \%$ reported in the Danish population, which has a similar age incidence rate and curve [14]. It is more likely that these differences are explained by geographical differences and the concomitant different treatment philosophy than by patient characteristics such as fracture complexity as both patient groups are similar [2,15]. The ROS was shaped like an inverse $U$ in both the Belgian population and at the UHL. The lower ROS in younger patients can be explained by the bone remodeling capacity and less displaced fractures, whereas the lower ROS in the elderly could be explained by less displaced fragility fractures and the perioperative risk. The ROS curves for patients treated in Belgium and at the UHL are similar. To our knowledge, this study is the first to report on the nationwide age dependent ROS for patients with a TPF.

Wasserstein et al. reported a 7.3\% 10-year likelihood ratio of a TKA after operative treatment of TPF [16]. This is slightly higher than $5 \%$ for operatively treated patients in Belgium and $4 \%$ at the UHL. As the magnitude of included patient in our study is more than tenfold less, we do not wish to withdraw hard conclusions from our results. Moreover, with regard to the TKA after TPF at the UHL, some patients might have got a TKA elsewhere and therefore are not included in our results.

Remarkably, $2 \%$ of the operatively treated TPF in Belgium were treated in ambulatory care, whereas none of the UHL patients. Wound care is the most obvious indication for this. Hypothetically patients could have been arthroscopically treated (e.g. tibial eminence fractures) and falsely recorded as TPF to the NIHIDI. After all tibial eminence fractures were not included in this study. Unfortunately, due to privacy regulations we could not gain insight in the patients records and therefore we are not able to provide a valid cause in this regard.

It is somewhat surprising that the LOS of non-operatively treated patients was longer than the LOS of operatively treated patients in Belgium (19.2 vs. 15.7 days, respectively). The authors believe that this 
supports the hypothesis that the increased number of non-operatively treated fractures might be due to higher comorbidity and increased perioperative risks. This indicates that there is often an underlying medical problem to the admission diagnosis of TPF, which requires a prolonged medical treatment by others.

The total healthcare costs for operatively and non-operatively treated TPF were similar and varied around $€ 9,500$. Hospitalization was identified as the most important driver of the total healthcare costs of both the operatively and non-operatively treated patients in Belgium and at the UHL. This is in line with previous studies wherein hospitalization (and honoraria) were identified as the main drivers of the total healthcare costs in the Belgian healthcare financing system $5,[7,17-18]$. In turn, the hospitalization costs are the result of the LOS multiplied by the national average day based care fee. Despite the lower hospitalization rate of non-operatively treated patients (but prolonged LOS in Belgium), the relative share of the hospitalization costs predominantly effects the total costs. Guidelines on appropriate LOS can help to decrease variability and curb the total healthcare costs. Note, of course this independent from the question which TPF should be operated and which should not $[16,19]$.

The implant related costs were relatively low due to the relatively high hospitalization costs. Although implant choice and number of implants used influences the cost for an individual patient, it did not significantly affect the total costs of TPF on the Belgian Health Care system.

With the continuing increase in healthcare costs and the rapid gaining importance of health economics, it seems justified to include the economic burden in decision-making about the surgical treatment of hip TPF fractures. However, since healthcare financing is country-specific, data and conclusions from this study cannot simply be compared linearly, without taking the differences in healthcare financing into account. The costs included in this study represent the hospital funds for patient care. Total reimbursements of hospitals takes place on a macro scale and is not bound by specific pathologies, as under- and overcompensation of pathologies balance out the total reimbursement, which can be seen as an approximation of the total costs of care for society.

Our study has a number of limitations. The first limitation is the nature of the study. It is a retrospective study and therefore it has inherent biases. Secondly we did not set any exclusion criteria as the database of the NIHDI is an insurance, cost-oriented database which links costs and a limited set of patient specific parameters (age, sex, location of residence) to a diagnosis. Therefore, patient related information such as comorbidities, past medical history, medication, etc. could not be obtained from this database. In order to create consistency between the NIHDI and UHL database, no exclusion criteria were set for the UHL database.

Furthermore, the database is country specific and therefore might not be applicable to other countries. However, our data shows that there was a relatively limited number of patients who needed a TKP following operative treatment. Also the cost of an operative treatment in Belgium is close to the cost of a nonoperative treatment as it might allow for an early active rehabilitation and a hospital stay which is as long as that of a non-operatively treated fracture.

In conclusion, there was an increase in the incidence of TPF in Belgium and these fractures are increasingly treated non-operatively. Although the hospitalization rate of these non-operatively patients was relatively low, their mean LOS was very long and increased over time. Since LOS is the main driver of the total healthcare costs in our cost model, this results in a cost that was almost equal for operatively and nonoperatively treated patients. Despite a higher hospitalization rate of nonoperatively treated TPF at the UHL (compared to Belgium), the LOS of non-operatively treated TPF was significantly lower compared to the operatively treated patients, and had significantly lower total healthcare cost per patient, subsequently. Therefore, it is of utmost importance that future studies look into LOS, particularly of non-operatively treated patients. Dedicated clinical care pathways as described for hip fractures could also be applicable for TPF and the inclusion of social services from the moment of admission are essential in order to have an appropriate discharge plan and therefore reduce the total healthcare costs.

\section{COMPLIANCE WITH ETHICAL STANDARDS}

- Disclosure of potential conflicts of interest 
- Research involving Human Participants

References

1. Frattini M, Vaienti E, Soncini G, Pogliacomi F. Tibial plateau fractures in elderly patients. Chir. Organi Mov. 2009;93:109-114.

2. Herteleer M, Hoekstra H, Nijs S. Diagnosis and treatment of clavicular fractures in Belgium between 2006 and 2015. J. Shoulder Elb. Surg. 2018;27:1512-1518.

3. Wennergren D, Bergdahl C, Ekelund J, Juto H, Sundfeldt M, Möller M. Epidemiology and incidence of tibia fractures in the Swedish Fracture Register. Injury. 2018;49:2068-2074.

4. van den Berg J, Reul M, Nunes Cardozo M, Starovoyt A, Geusens E, Nijs S, Hoekstra H. Functional outcome of intra-articular tibial plateau fractures: the impact of posterior column fractures. Int. Orthop. 2017;41:1865-1873.

5. Metsemakers WJ, Smeets B, Nijs S, Hoekstra H. Infection after fracture fixation of the tibia: Analysis of healthcare utilization and related costs. Injury. 2017;48:1204-1210.

6. Oladeji L, Worley J, Crist B. Age-Related Variances in Patients with Tibial Plateau Fractures. J. Knee Surg. 2019.

7. Herteleer M, De Jaegere A, Winckelmans T, Casteur H, Nijs S, Hoekstra H. Healthcare utilization and related cost of midshaft clavicle fracture treatment in Belgium. Eur. J. Trauma Emerg. Surg. 2020.

8. Belgian Governement. RIZIV - INAMI - NIHDI. 2017. Available at: http://www.inami.fgov.be.

9. Statistics BDG. Population Belgium. 2017. Available at: http://statbel.fgov.be/nl/statistieken/cijfers/bevolking/structuur/leeftijdgeslacht/belgie/. Data retrieved in October 2019

10. Gaunder CL, Zhao Z, Henderson C, McKinney BR, Stahel PF, Zelle BA. Wound complications after open reduction and internal fixation of tibial plateau fractures in the elderly: a multicentre study. Int. Orthop. 2019;43:461-465.

11. Morris BJ, Unger RZ, Archer KR, Mathis SL, Perdue AM, Obremskey WT. Risk Factors of Infection After ORIF of Bicondylar Tibial Plateau Fractures. J. Orthop. Trauma. 2013;27:e196-e200.

12. Court-Brown CM, Caesar B. Epidemiology of adult fractures: A review. Injury. 2006;37:691-697. 13. Leroux T, Wasserstein D, Henry P, Khoshbin A, Dwyer T, Ogilvie-Harris D, Mahomed N, Veillette C. Rate of and Risk Factors for Reoperations After Open Reduction and Internal Fixation of Midshaft Clavicle Fractures: A Population-Based Study in Ontario, Canada. J. Bone Joint Surg. Am. 2014;96:1119-1125.

13. Vestergaard V, Pedersen AB, Tengberg PT, Troelsen A, Schrøder HM. 20-year trends of distal femoral, patellar, and proximal tibial fractures: a Danish nationwide cohort study of 60,823 patients. Acta Orthop. 2020;91:109-114.

14. Du Bois M, Szpalski M, Donceel P. A decade's experience in lumbar spine surgery in Belgium: Sickness fund beneficiaries, 2000-2009. Eur. Spine J. 2012;21:2693-2703.

15. Wasserstein D, Henry P, Paterson JM, Kreder HJ, Jenkinson R. Risk of total knee arthroplasty after operatively treated tibial plateau fracture a matched-population-based cohort study. J. Bone Jt. Surg-Ser. A. 2014;96:144-150.

16. Hoekstra H, Smeets B, Metsemakers WJ, Spitz AC, Nijs S. Economics of open tibial fractures: the pivotal role of length-of-stay and infection. Health Econ. Rev. 2017;7.

17. Metsemakers WJ, Smeets B, Nijs S, Hoekstra H. Infection after fracture fixation of the tibia: Analysis of healthcare utilization and related costs. Injury. 2017;48:1204-1210.

18. Monteban P, van den Berg J, van Hees J, Nijs S, Hoekstra H. The outcome of proximal fifth metatarsal fractures: redefining treatment strategies. Eur. J. Trauma Emerg. Surg. 2018;44:727-734.

19. Garrison Kimberly R, Shemilt I, Donell S, Ryder Jonathan J, Mugford M, Harvey I, Song F, Alt V. Bone morphogenetic protein (BMP) for fracture healing in adults. Cochrane Database Syst. Rev. 2010. 


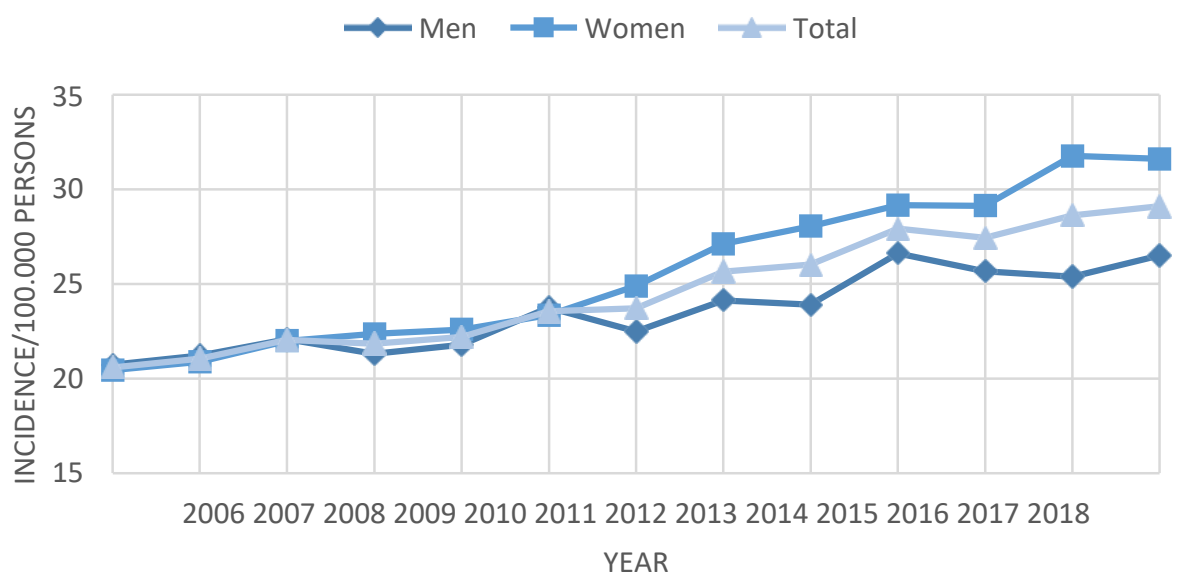

Figure 1. Incidence of TPF in Belgium between 2006 and 2018 


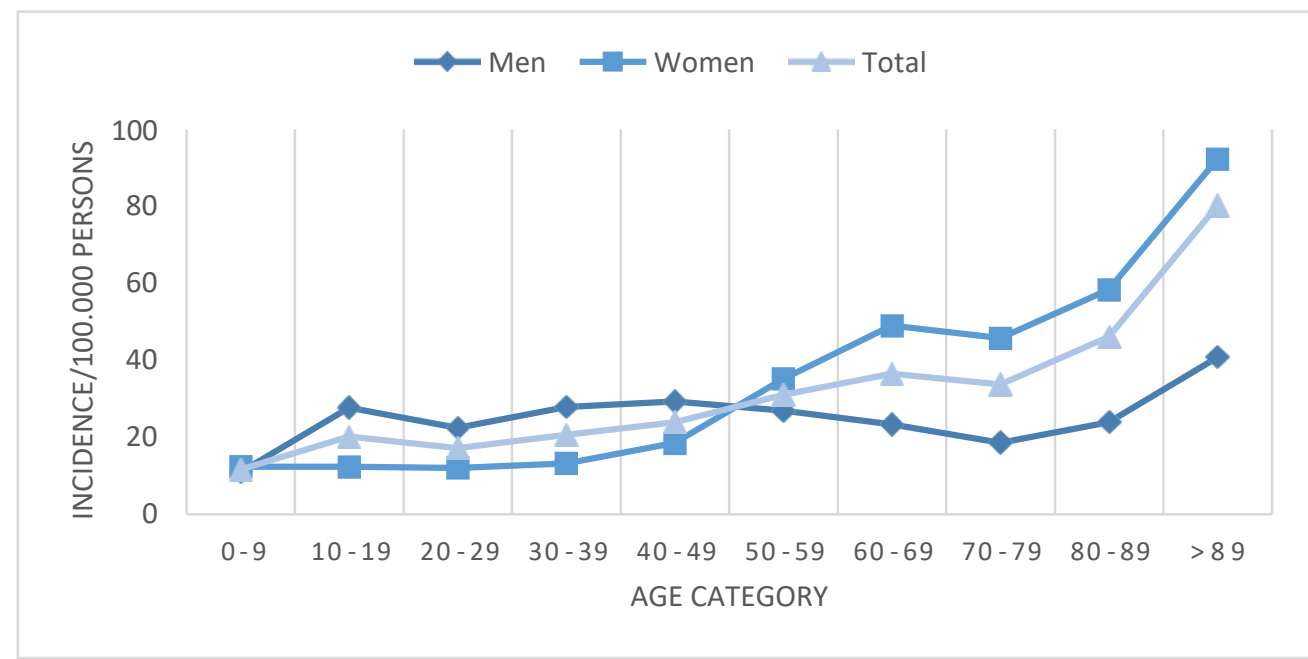

Figure 2: Age adjusted incidence of TPF in Belgium 

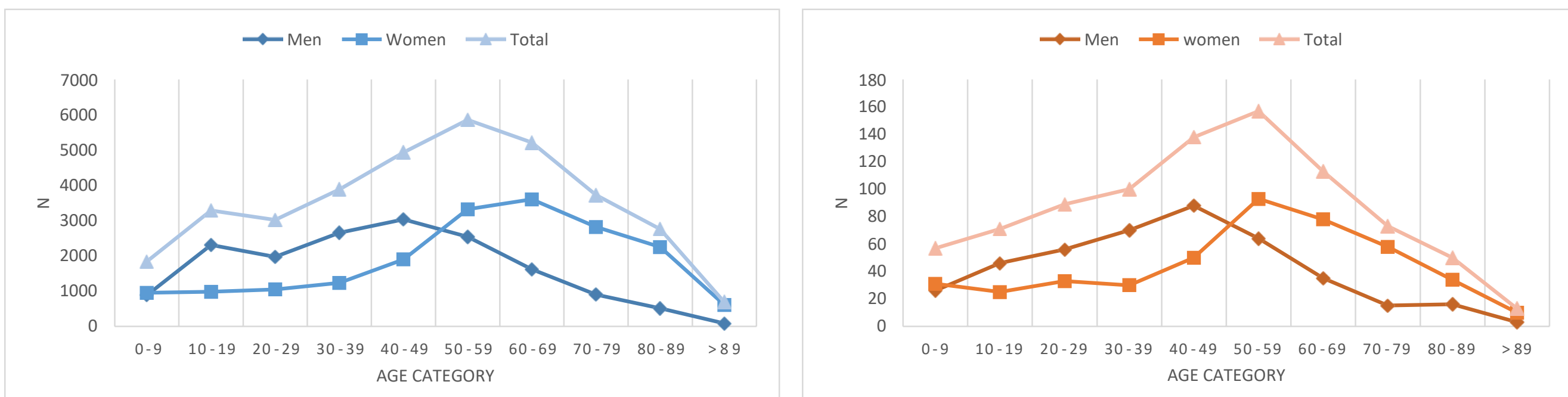

Figure 3: Absolute number of TPF in Belgium (A) and UHL (B) between 2006 and 2018 

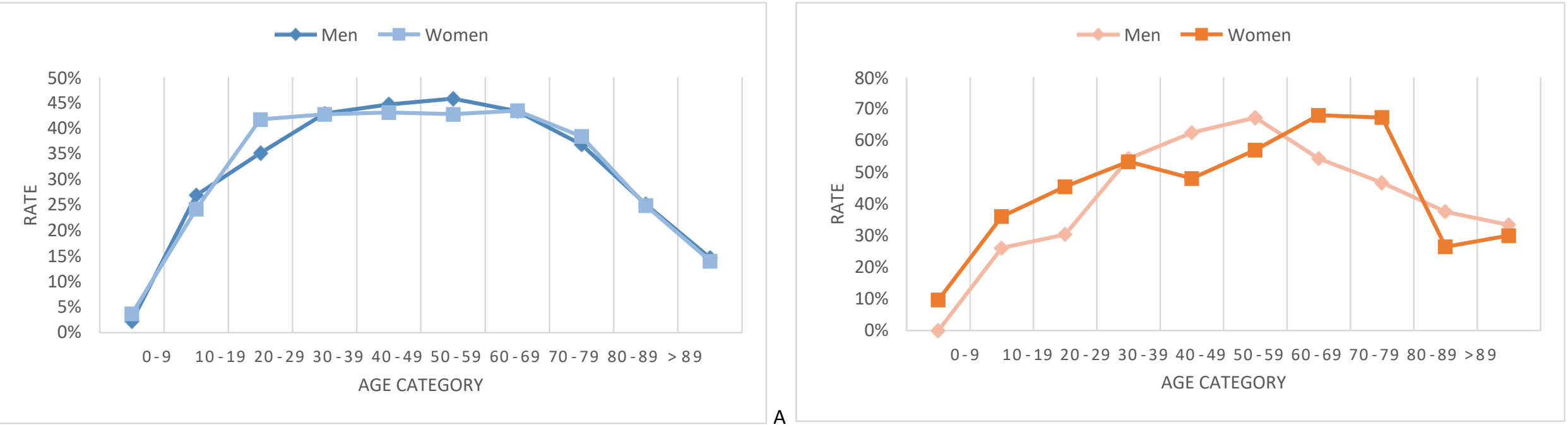

Figure 4: Rate of surgery related to age cluster in Belgium (A) and UHL (B). 

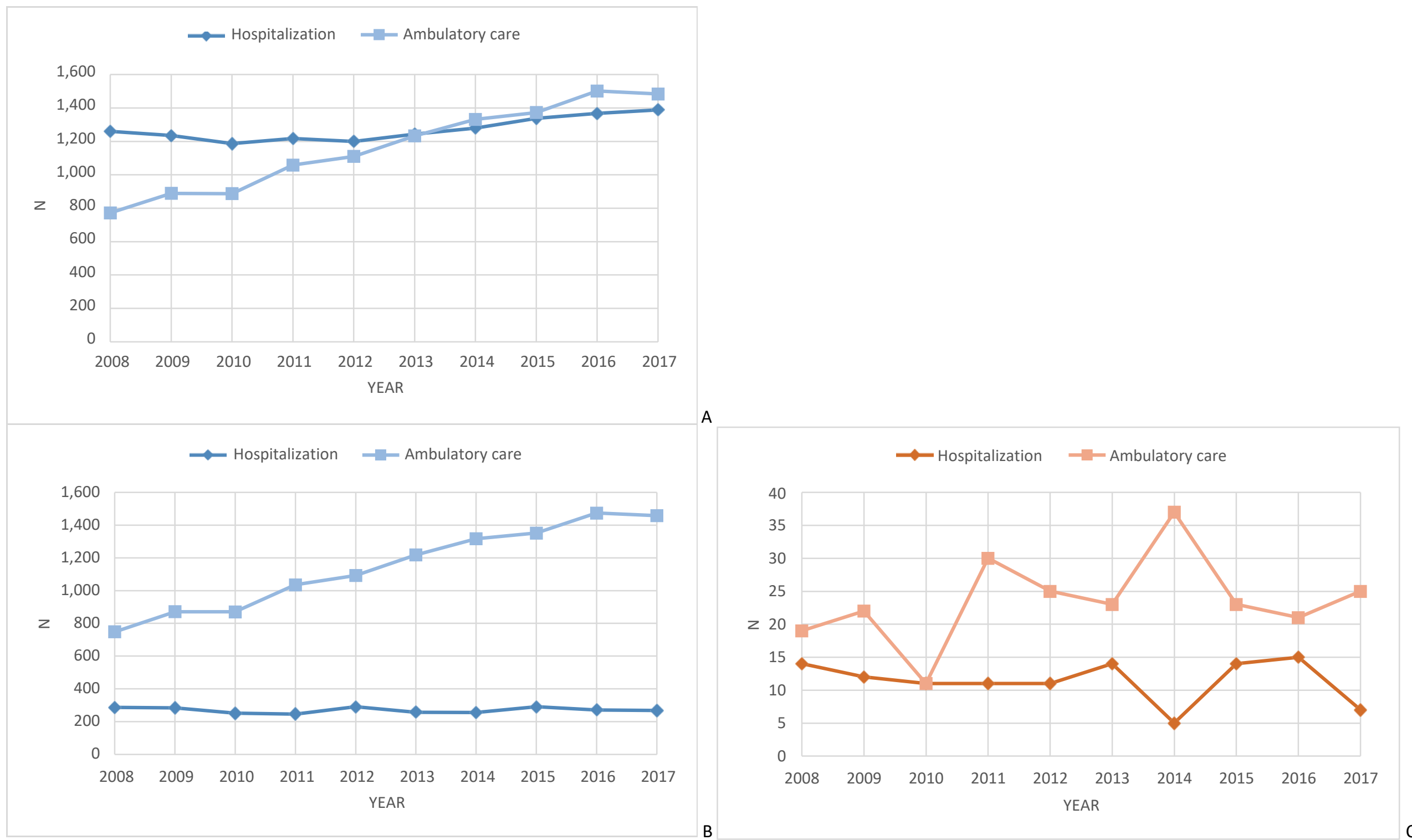

Figure 5: hospitalization and day care for (all) TPF in Belgium (A); hospitalization and day care for non-operatively treated TPF in Belgium (B); hospitalization and day care for nonoperatively treated TPF at the UHL 

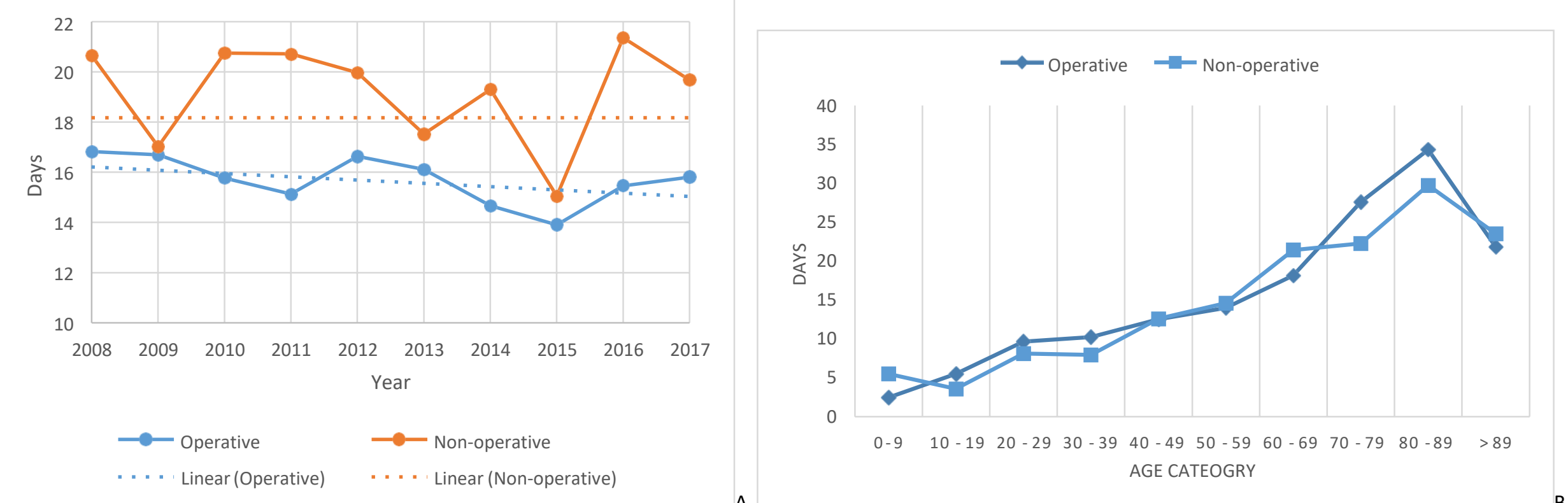

Figure 6: Mean LOS of operatively and non-operatively treated TPF in Belgium (A); Mean LOS for Belgian inpatients with TPF per age category between 2008 and 2017 (B) 


\begin{tabular}{|c|c|c|c|c|c|c|c|}
\hline & & \multicolumn{3}{|c|}{ Belgium } & \multicolumn{3}{|c|}{ UHL } \\
\hline & & Operative & Non-operative & Total (n\%) & Operative & Non-operative & Total $(\mathrm{n} \%)$ \\
\hline \multirow[t]{3}{*}{ Epidemiology } & Male & $6,162(37 \%)$ & $10,344(63 \%)$ & $16,506(100 \%)$ & $198(47 \%)$ & $221(53 \%)$ & $419(100 \%)$ \\
\hline & Female & $6,765(36 \%)$ & 11,955 (64\%) & $18,720(100 \%)$ & $224(51 \%)$ & $218(49 \%)$ & $442(100 \%)$ \\
\hline & Total & $12,927(37 \%)$ & $22,299(63 \%)$ & $35,226(100 \%)$ & $422(49 \%)$ & $439(51 \%)$ & $861(100 \%)$ \\
\hline
\end{tabular}

Table 1. Number of TPF per sex in Belgium and at the UHL between 2006 and 2018 


\begin{tabular}{|c|c|c|c|c|c|c|c|}
\hline & & \multicolumn{3}{|c|}{ Belgium } & \multicolumn{3}{|c|}{ UHL } \\
\hline & & Operative & Non-operative & Total & Operative & Non-operative & \\
\hline \multirow[t]{3}{*}{ Healthcare utilization, number of } & Hospitalization & 10,012 (98\%) & $2,698(19 \%)$ & $12,710(52 \%)$ & $300(100 \%)$ & $114(32 \%)$ & $414(63 \%)$ \\
\hline & Ambulatory care & $197(2 \%)$ & $11,434(81 \%)$ & $11,631(48 \%)$ & 0 & $245(68 \%)$ & $245(37 \%)$ \\
\hline & Total & $10,209(100 \%)$ & $14,132(100 \%)$ & 24,341 (100\%) & $300(100 \%)$ & 359 (100\%) & 659 (100\%) \\
\hline LOS (days) & LOS & $15.7(1-465)$ & $19.2(1-626)$ & & $18.6(1-852)$ & $12.8(1-279)$ & $P<0.001$ \\
\hline \multirow[t]{2}{*}{ Total Healthcare costs } & Hospitalization & $€ 9,481( \pm 483)$ & $€ 9,838( \pm 692)$ & & $€ 10,110( \pm 3,703)$ & $€ 8,543( \pm 3,789)$ & $P<0.001$ \\
\hline & Ambulatory care & $€ 175( \pm 50)$ & $€ 32( \pm 2)$ & & - & $€ 264( \pm 37)$ & $P<0.001$ \\
\hline
\end{tabular}

Table 2: Healthcare utilization, mean LOS (range) and mean total healthcare cost ( \pm SD) per patient in Belgium and at the UHL between $2008-2017$ 
